\title{
Crashworthiness optimization of bionic bumper structure under low-speed impact
}

\author{
Tao $\mathrm{Xu}^{1}$, Nian $\mathrm{Liu}^{1}$, Yuan $\mathrm{Tian}^{2}$, Tianshuang $\mathrm{Xu}^{1}$, Tianyi Zhang ${ }^{1}$, Zhenglei $\mathrm{Yu}^{3, *}$, and Yiwen \\ $\mathrm{Li}^{4}$ \\ ${ }^{1}$ School of Mechanical Science and Engineering, Jilin University, 130022 Changchun, China \\ ${ }^{2}$ Control Engineering, Air Force Aviator University, 130022 Changchun, China \\ ${ }^{3}$ Key Lab of Bionic Engineering, Ministry of Education, Jilin University, 130022 Changchun, China \\ ${ }^{4} \mathrm{R} \&$ D Center, China FAW Co., Ltd, 130011 Changchun, China
}

\begin{abstract}
By considering the crashworthiness design of bionic bumper structure during frontal impact, the thicknesses are chosen to analysis and optimal design to obtain the lightweight demand. The orthogonal experiment design and radial basis function are employed to construct the response surface for the performances of thin-walled components. The multi-objective cuckoo search (MOCS) is applied to perform the optimal design. The results demonstrate that the optimal method and process proposed have high accuracy and validity.
\end{abstract}

\section{Introduction}

In the field of the passive safety of passenger vehicles, the studies of crush stiffness characteristics are very important. In order to reduce the damage to occupants in a collision, it is necessary to maximize the energy absorption of frontal components. The safety demands cause an increase in weight of traditional vehicle body. In nature, some creatures have good mechanical properties as well as lightweight structures, it is feasible to apply biological methods to energy-absorbing structures, which can ensure the lightweight design while improving the energy absorption capacity [1,2].

To seek for optimal crashworthiness of structure, nonlinear finite element method has been extensively employed. Unfortunately, high nonlinearities in numerical sensitivity analysis could to a certain extent compromise the efficiency and feasibility of the conventional FE-based gradient optimization procedures [3, 4]. Therefore, a surrogate model technique is represented by the response surface method (RSM) to solve highly nonlinear problem, especially in bionic structure design $[5,6]$.

This work aims at designing the appropriate configuration of frontal bumper structure based on bionic method. According to our previous research, the bionic bumper structure (bio-crash beam and bio-box, B-CB\&B) is used as the basic model. The material thickness needs to be optimized to achieve the passive safety target. Meanwhile, lightweight design must be considered. Set up experiments by Orthogonal experiment, then the RSM based on radial basis function method is adopted to construct the functions of the specific energy

\footnotetext{
* Corresponding author: zlyu@jlu.edu.cn
} 
absorption (SEA) with respect to the components' thicknesses. Finally, by using the multiobjective cuckoo search (MOCS), the optimal thickness parameters of the components are obtained to minimize the weight.

\section{Description of optimization problem}

For the frontal crash scenario, the main function of a bumper structure is to attenuate the impact of a collision in direct contact [7]. Figure 1 shows the initial bumper structure. In nature, cattail and bamboo have good dynamic mechanical properties when bearing external pressure. Inspired by their ingenious structures, we designed several types of the bionic energy box applied to bumper. Particularly, the B-CB\&B type bionic structure has excellent energy absorption characteristics $[8,9]$, and it is worth to pay more study on the optimization problem, as shown in Figure 2.

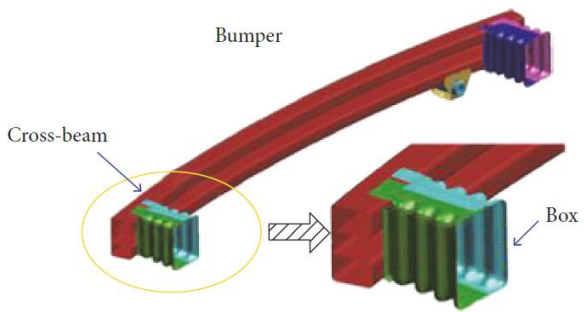

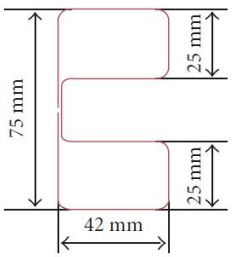

Cross-beam section

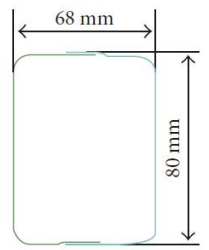

Box section

Fig. 1. Initial bumper structure.

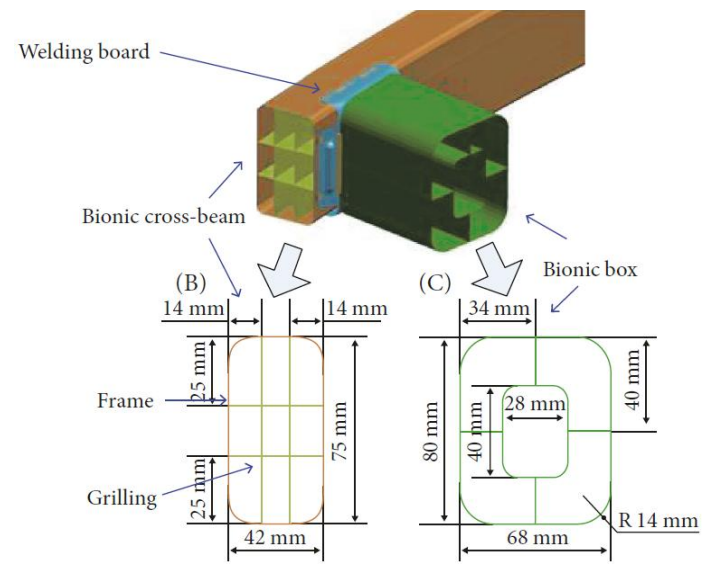

Fig. 2. Bionic bumper structure.

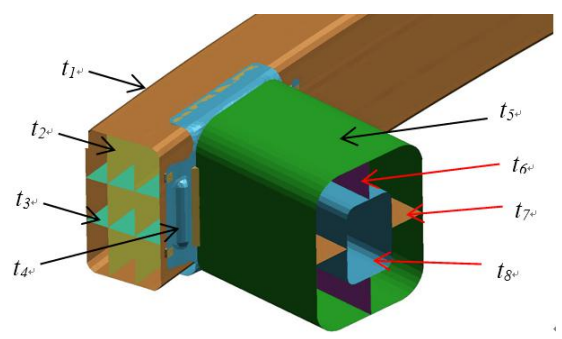

Fig. 3. 8 design variables of bionic bumper structure.

The inner reinforcement components and load-bearing assembles could not be removed due to collision stability demands. The materials of components will also not change. 
Therefore, choosing the thicknesses of 8 key components (displays in Figure 3) as design variables to reach the lightweight target under crashworthiness constraints. The optimization problem can be expressed as in equation (1).

$$
\begin{aligned}
& \text { Min } F_{\text {max }} \\
& \text { s.t. } \quad S E A \geq 2.592 \mathrm{~kJ} / \mathrm{kg} \\
& t_{1} \in[1.5 \mathrm{~mm}, 2.5 \mathrm{~mm}] \\
& t_{2}, t_{3} \sim t_{8} \in[1.5 \mathrm{~mm}, 2 \mathrm{~mm}] \\
& S E A=\text { Total energy absorption/Total mass }
\end{aligned}
$$

where $S E A$ is specific energy absorption of total components, it denotes the energy absorbed per unit mass of absorber as in equation (2), which is used to estimate the energy absorption characteristics of safety protection equipment. The $F_{\max }$ represents the peak section force of rear domain on bionic box, and it must be controlled to keep the loading path continuity and stability. Therefore, the objective is to minimize $F_{\max }$. The SEA of initial B-CB\&B type bionic structure is $2.592 \mathrm{~kJ} / \mathrm{kg}$ and that value is set to be the lower limit. $t_{1}, t_{2} \sim t_{8}$ represent the thickness variables of 8 components as shown in figure 3 .

The simulation scenario we used here is a full-size rigid wall collision as shown in figure 4. By calculating kinetic energy conservation, the weight of rigid wall is $930 \mathrm{~kg}$. The initial crash speed is set to $4.33 \mathrm{~m} / \mathrm{s}$. Table 1 shows material and thickness information of initial bionic structure. The nonlinear analysis is carried out using LS-DYNA.

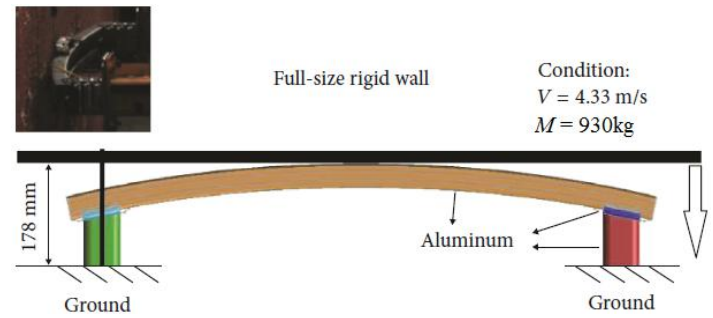

Fig. 4. Full-size crash condition.

Table 1. The parameters of initial bionic structure.

\begin{tabular}{|c|c|c|c|}
\hline Component & Part & Material & Thickness (mm) \\
\hline \multirow{2}{*}{ Crossbeam } & Frame & Extrusion aluminium (6062T6) & 2.0 \\
\cline { 2 - 4 } & Grilling & Extrusion aluminium (6062T6) & 1.7 \\
\hline Box & - & Extrusion aluminium (6062T6) & 1.7 \\
\hline Welding plate & - & High-strength steel (st280) & 1.4 \\
\hline
\end{tabular}

\section{Surrogate Model by RSM}

RSM is a collection of mathematical and statistical techniques for constructing global approximations assessment based on objective and constraint functions in various samples in the design space.

\subsection{Definition of Design Point}


Design of experiment (DOE) provides a means to the selection of the samplings in the design domain, and the basic aim of this method is to maximize design efficiency and accuracy [10]. By considering the accuracy and computing cost, two-level orthogonal design table $L_{16}\left(2^{15}\right)$ of 8 variables $\left(t_{1}, t_{2}, \cdots, t_{8}\right)$ is chosen [11], then the analysis details are listed in table 2 .

Table 2. Design samples and responses.

\begin{tabular}{|c|c|c|c|c|c|c|c|c|c|c|}
\hline \multirow{2}{*}{$\begin{array}{l}\text { Design } \\
\text { number }\end{array}$} & \multicolumn{7}{|c|}{$t_{i}(\mathrm{~mm})$} & \multirow{2}{*}{$S E A(\mathrm{~kJ} / \mathrm{kg})$} & \multirow{2}{*}{$F_{\max }(\mathrm{kN})$} \\
\cline { 2 - 10 } & $t_{1}$ & $t_{2}$ & $t_{3}$ & $t_{4}$ & $t_{5}$ & $t_{6}$ & $t_{7}$ & $t_{8}$ & & \\
\hline 1 & 1.5 & 1.5 & 1.5 & 1.5 & 1.5 & 1.5 & 1.5 & 1.5 & 3.031 & 138.67 \\
\hline 2 & 2.5 & 1.5 & 2 & 1.5 & 2 & 1.5 & 2 & 1.5 & 2.292 & 122.93 \\
\hline 3 & 1.5 & 2 & 2 & 1.5 & 1.5 & 2 & 2 & 1.5 & 2.707 & 80.61 \\
\hline 4 & 2.5 & 2 & 1.5 & 1.5 & 2 & 2 & 1.5 & 1.5 & 2.247 & 111.39 \\
\hline 5 & 1.5 & 1.5 & 1.5 & 2 & 2 & 2 & 2 & 1.5 & 2.819 & 115.94 \\
\hline 6 & 2.5 & 1.5 & 2 & 2 & 1.5 & 2 & 1.5 & 1.5 & 2.357 & 71.08 \\
\hline 7 & 1.5 & 2 & 2 & 2 & 2 & 1.5 & 1.5 & 1.5 & 2.537 & 107.14 \\
\hline 8 & 2.5 & 2 & 1.5 & 2 & 1.5 & 1.5 & 2 & 1.5 & 2.256 & 66.23 \\
\hline 9 & 1.5 & 1.5 & 1.5 & 1.5 & 1.5 & 1.5 & 1.5 & 2 & 2.942 & 63.16 \\
\hline 10 & 2.5 & 1.5 & 2 & 1.5 & 2 & 1.5 & 2 & 2 & 2.277 & 120.72 \\
\hline 11 & 1.5 & 2 & 2 & 1.5 & 1.5 & 2 & 2 & 2 & 2.621 & 77.39 \\
\hline 12 & 2.5 & 2 & 1.5 & 1.5 & 2 & 2 & 1.5 & 2 & 2.183 & 110.15 \\
\hline 13 & 1.5 & 1.5 & 1.5 & 2 & 2 & 2 & 2 & 2 & 2.785 & 105.42 \\
\hline 14 & 2.5 & 1.5 & 2 & 2 & 1.5 & 2 & 1.5 & 2 & 2.292 & 67.94 \\
\hline 15 & 1.5 & 2 & 2 & 2 & 2 & 1.5 & 1.5 & 2 & 2.508 & 99.61 \\
\hline 16 & 2.5 & 2 & 1.5 & 2 & 1.5 & 1.5 & 2 & 2 & 2.225 & 54.32 \\
\hline
\end{tabular}

\subsection{Construction of multi-parameters RSM}

In this paper, second-order formula (as equation (3)) is employed to describe the relationships between the responses and design factors. As a result of the approximated modelling method based on radial basis function, the quadratic response functions of $S E A$ and linear function of $F_{\max }$ are obtained.

$$
\hat{y}=a+\sum_{i=1}^{m} b_{i} x_{i}+\sum_{i=1}^{m} b_{i i} x_{i}^{2}+\sum_{i<k}^{m} b_{i k} x_{i} x_{k} .
$$

Then, based on the RS models and FE analysis results, calculate the correlation coefficients $R \in[0,1]$ [11]. The $R \rightarrow 1$ represents a good fitness between initial model and surrogate model. Otherwise, the samples must be re-chosen. The $R$ of the quadratic response function of $\delta_{\text {in }}$ equals to 1 . It means the surrogate model is adequate to fit the response and it is feasible to execute the optimal design.

\section{Optimal design by MOCS}

The multi-objective cuckoo search (MOCS) is a heuristic evolutionary algorithm inspired by cuckoo's breed behaviour [12] which has the advantages of simple structure, less parameters and superior search path [13]. It updates the solution using the Lévy flight mechanism in the optimization process, the search path and location update formula is defined as in equation (4)-(6):

$$
x_{i}^{(t+1)}=x_{i}^{(t)}+\alpha \oplus \operatorname{Lé} v y(\beta)
$$




$$
\begin{gathered}
\alpha=\alpha_{0}\left(x_{j}{ }^{(t)}-x_{i}{ }^{(t)}\right) \\
L \text { évy } \sim u=t^{-1-\beta}(0 \leq \beta \leq 2)
\end{gathered}
$$

$x_{i}^{(t+1)}$ is the $i$ cuckoo individual in the $(t+1)$ generation, $\oplus$ is entry-wise multiplications, $\alpha$ is the step size, $\alpha_{0}$ is constant to control the step size. $\operatorname{Le} v y(\beta)$ is the random step which is drawn from a Lévy distribution for large steps. The algorithm flow chart shows in figure 5. Table 3 lists the optimal thicknesses of the components by MOCS. Also the weight and intrusion were calculated by crash analysis. For the manufacturing factors, the optimal thicknesses have to be round-off.

\begin{tabular}{|c|c|c|c|c|c|c|c|c|c|c|}
\hline \multirow{2}{*}{$\begin{array}{l}\text { Design } \\
\text { number }\end{array}$} & \multicolumn{8}{|c|}{$t_{i}(\mathrm{~mm})$} & \multirow{2}{*}{$\begin{array}{r}S E A \\
(\mathrm{~kJ} / \mathrm{kg})\end{array}$} & \multirow{2}{*}{$\begin{array}{c}F_{\max } \\
(\mathrm{kN})\end{array}$} \\
\hline & $t_{1}$ & $t_{2}$ & $t_{3}$ & $t_{4}$ & $t_{5}$ & $t_{6}$ & $t_{7}$ & $t_{8}$ & & \\
\hline Initial model & 2.0 & 1.7 & 1.7 & 1.4 & 1.7 & 1.7 & 1.7 & 1.7 & 2.592 & 151.72 \\
\hline Optimal model & 1.51 & 1.50 & 1.50 & 1.52 & 1.53 & 1.61 & 1.57 & 1.63 & 2.964 & 63.89 \\
\hline Round-off model & 1.5 & 1.5 & 1.5 & 1.5 & 1.5 & 1.6 & 1.6 & 1.6 & 2.982 & 64.52 \\
\hline
\end{tabular}

Table 3. Results comparison between initial and optimal model.

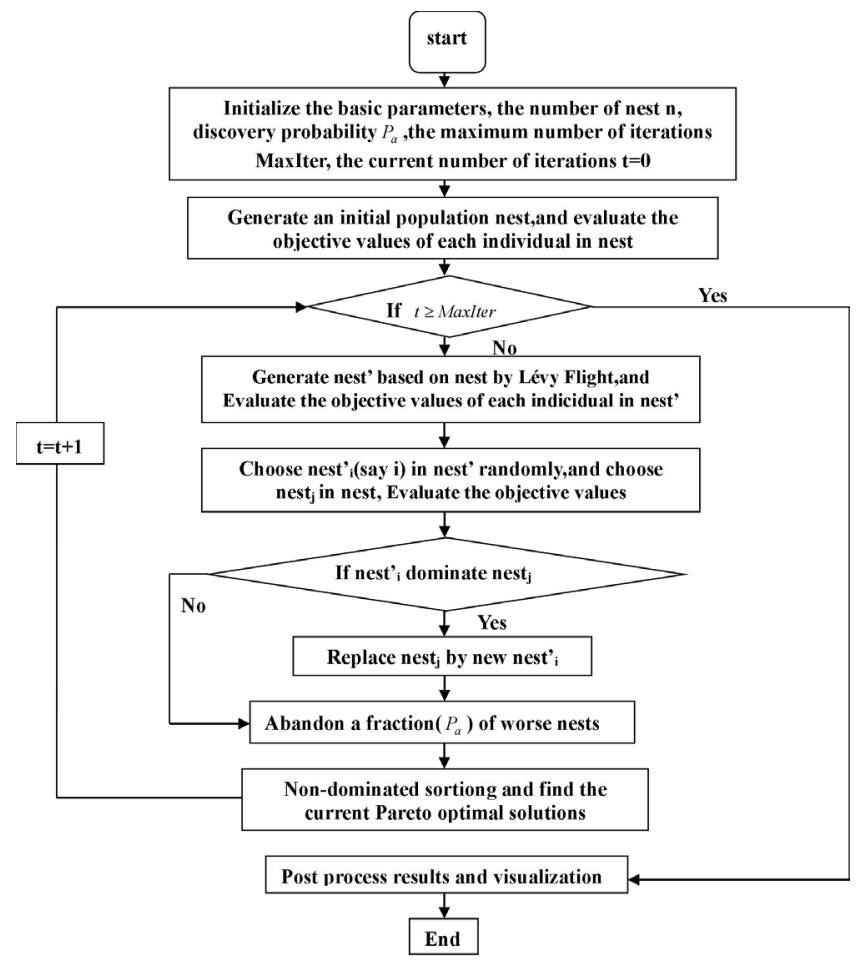

Fig. 5. The flow chart of MOCS.

Based on round-off results, the peak section force of bionic box at rear domain reduces nearly $57.5 \%$. Considering special limitations of the protection frame design, the optimal effect is completely obvious by only changing the components' thicknesses. Meanwhile, the SEA increases remarkably and it does satisfy the constraint. Therefore, the optimization method used in this paper is very effective. Based on the optimization, the sensitivity analysis could not be performed because of nonlinear problem, but optimal thickness 
distribution shows that the significantly different influence of the whole characteristics by different components.

\section{Conclusions}

(1) In order to improve the crashworthiness performance of the frontal protection components, DOE is implied to construct the RS models with high accuracy. Therefore, the approximate sensitivities, or variation trend of responses with regards to thicknesses can be observed from the DOE results.

(2) Based on the RS functions and MOCS, find the optimal thickness distribution directly. Consider the lightweight demands, energy absorption and peak crash force simultaneously. Therefore, the optimal bionic bumper structure is preferable by comprehensively measurement.

(3) Due to the limitations from manufacturing, it is clear that the engineers gain a great benefit on the cost and timing by utilizing the DOE and optimization approach to improve the crashworthiness of bionic structure. The DOE and MOCS applied in this paper are worth to be better used in modifying the structural crashworthiness characteristics under the other conditions.

\section{Acknowledgments}

The authors would like to acknowledge the National Natural Science Funds (Grant No. 51505181 and 51405186), the China Postdoctoral Science Foundation funded project (No. 2016M590256), the Advanced Manufacturing Project of Provincial School Construction of Jilin Province (No. SXGJSF2017-2).

\section{References}

1. Zou M, Xu S C, Wei C G, Wang H X and Liu Z Z (2016) Thin. Wall. Struct 101222 30

2. Zhao Z L, Huang W X, Li B W, Chen K X, Chen K F, Zhao H P and Feng X Q (2015) J. Bionic. Eng. 12 634-42

3. Liu Y C (2008) Thin. Wall. Struct 46 1329-37

4. Forsberg J and Nilsson L (2006) Int. J. Impact. Eng 32 759-77

5. Li Y W, Xu T, Xu T S, Li Q and Hao L (2010) T. B. Inst. Technol. 30 1175-9

6. Zhang Y, Li G Y and Zhong Z H (2008) Chin. J. Mech. Engen. 44 192-6

7. Zarei H and Kroger M (2008) Mater Des 28 193-204

8. Zou M, Wei C G, Li J Q, Xu S C and Zhang X (2015) Thin. Wall. Struc 95 255-61

9. Xu T, Liu N, Yu Z L, Xu T S and Zou M (2017) Appl. Bionics. Biomech. 2017 1-9

10. Hao L, Xu T, Cui J and Yoshino T (2013) J. JLU. (Engineering Technology Edition) 39 12-6

11. Montgomery D C (2006) Design and Analysis of experiments(New York: John Wiley \& Sons)

12. Yang X S and Deb S (2011) Comput. Oper. Res. 40 1616-24

13. Layeb A, Lahouesna $\mathrm{N}$ and Kireche B (2013) Int. J. Inform. Engine. El. Bus. 5 8-15 